\title{
Bestimmung und Deutung von Angebotskurven Ein Beispiel
}

\author{
Von
}

\section{J. Tinbergen, Scheveningen}

§1. Den quantitativen Bestimmungen von Nachfrage- und Angebotskurven, wie sie in der letzten Zeit mehrfach versucht worden sind, liegt im allgemeinen die folgende ,einfache statische Theorie des Marktes" (das "Schema von Walras") zugrunde: Es sei $\pi$ irgendein denkbarer (virtueller) Preis; die gesamte Nachfrage, welche bei diesem Preise besteht, sei $n(\pi)$, das gesamte Angebot $a(\pi)$. Dann wird der wirklich eintreffende (reelle) Preis $p$ durch die Gleichung

$$
a(p)=n(p)
$$

bestimmt, während die wirklich stattfindende Nachfrage bzw. das wirklich stattfindende Angebot (der Umsatz) $u$ der Gleichung $u=$ $a(p)=n(p)$ gehorcht (Abb. I).

Das Problem der Bestimmung der Nach-

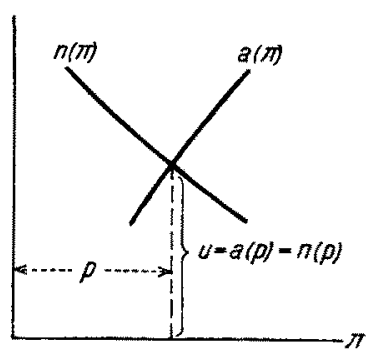

Abb. 1 frage- und Angebotskurven ist gerade das "umgekehrte" dieser Theorie und lautet also generell: gegeben sind $p$ und $u$ als Funktionen der Zeit, gesucht sind die Funktionen $a(\pi)$ und $n(\pi)$.

Im einfachsten denkbaren Fall, wo $a$ und $n$ außer $\pi$ nur noch Konstanten enthalten, und wo infolgedessen $p$ und $u$ beide anch Konstanten sind, ist diese Aufgabe ohne weitere Daten unlösbar.

Meistens liegen die Verhältnisse aber anders. Entweder $a$ oder $n$ oder auch beide enthalten neben $\pi$ einen oder mehrere Parameter, welche mit der Zeit veränderlich sind. Dadurch werden auch $p$ und $u$ variabel. Stellt man sich dann die Aufgabe, aus $p(t)$ und $u(t) a(\pi)$ und $n(\pi)$ aufzufinden, so ist diese wieder unlösbar obne weitere Angaben über die Parameter ${ }^{1}$ ).

Lösbar wird die Aufgabe teilweise oder ganz, wenn gegeben ist, von welchen Parametern $a$ oder $n$ noch abhängen und wie diese Parameter verlaufen. Der Einfachheit halber und in Utbereinstimmung mit der bisherigen Praxis werden wir bei den folgenden diesbezüglichen Ausführungen annehmen, daß $a$ sowie $n$ in $\pi$ und in den Parametern linear sind.

1) Auf wahrscheinlichkeitstheoretischer Basis wird dieses jedoch geleistet durch die geistreiche Methode Leontieffs (Weltw. Archiv, S. 1*, Juli 1929), welche aber für die hier gesetzten Ziele nicht weiter in Betracht kommt. 
\$ 2. Als nächsteinfacher, zweiter, Fall ist dann derjenige zu betrachten, wo nur $a$ noch einen Parameter $A$ enthält, so daß

$$
a=a_{0}+a_{1} \pi+a_{2} A, \quad n=n_{0}+n_{1} \pi
$$

Es ist dann leicht einzusehen, daß man $n_{1}$ bestimmen kann aus $u(t), A(t)$ und $p(t), a_{1}$ aber nicht. Es ist nämlich, wenn wir die $\pi$ und $A$ so messen, $\mathrm{da} B$ ihr Mittelwert $=0$ ist, und weil dann also $a_{0}=n_{0}$ ist,

d. h.

$$
\begin{gathered}
a_{1} p+a_{2} A=n_{1} p \\
p=\frac{a_{2}}{n_{1}-a_{1}} A,
\end{gathered}
$$

also $p$ mit $A$ proportional. Statistisch läßt sich dann nicht feststellen, "welcher Teil der Bewegungen von $a$ herstammt, von $p$ und welcher von $A^{\text {" }}$, d. h. man findet nur $n_{1}$ und eine nicht weiter zu analysierende Kombination von $a_{1}$ und $a_{2}$; es ist nämlich

$$
u=n_{0}+\frac{n_{1} a_{2}}{n_{1}-a_{1}} A \quad p=\frac{a_{2}}{n_{1}-a_{1}} A
$$

woraus $n_{1}$ und $\frac{a_{2}}{n_{1}-a_{1}}$ bestimmbar sind.

Dieser Fall ist schon mehrfach in statistischen Untersuchungen aufgetreten: die meisten landwirtschaftlichen Preise gehören mehr oder weniger deutlich hierher. Der Parameter $A$ ist hier die Ernte ${ }^{1}$ ). Man kann hier also nur die Nachfragekurve bestimmen; im Fall, daß nur in $n$ ein Parameter auftritt (wie angenähert bei Eisen der Fall sein dürfte), kann man nur die Angebotskurve bestimmen.

Diese Schwierigkeit ist von Moore ${ }^{2}$ ) und auch von Schultz in dieser Weise gelöst worden, daß angenommen wurde, es bestehe zwischen $a$ und $\pi$ kein gleichzeitiger Zusammenhang, sondern ein verzögerter. Wenn man die Zeitpunkte (Periodenmittelwerte) durch den oberen Index angibt:

während doch auch $a^{(t)}=n^{(t)}$ gesetzt wird.

$$
a^{(t)}=a_{0}+a_{1} \pi^{(t-1)}
$$

Dieses trifft aber nur dann zu, wenn wirklich nur der Vorjahrspreis für das Angebot bestimmend ist und nicht auch ein Naturfaktor hinzutritt, wie das doch bei den meisten landwirtschaftlichen Produkten bestimmt der Fall ist. Weiter sieht man an Hand der mit Abb. 1 analogen $\mathrm{Abb} .2$ leicht ein, daß nur für Fälle, wo angenähert $a_{1}=-n_{1}$ ist, eine bleibende Bewegung der Preise und Umsätze möglich ist. Sobald $a_{1}$ von $-n_{1}$ stark abweicht, führt der hier angegebene Mechanismus entweder zu immer stärker werdenden Schwankungen oder zur schnellen Herstellung einer Gleichgewichtslage (Abb. 2a bzw. 2b).

1) Vgl. z. B. Moore: A moving Equilibrium of Demand and Supply. S. 357. Quart. journ. of Econ., 1925, und viele andere; in letzter Zeit noch Donner: Bestimmungsgründe der Baumwollpreise. Sonderheft 15. z. d. Vjh. zur Konjunkturforsch. 1930; Nederlandsche Conjunctuurlijnen, 3. Viertelj., S. $17 \mathrm{ff}$. (85ff.) 1929.

2) Moore: a. a. 0 .

s) Schultz: Statistical Laws of Demand and Supply. Chicago. 1928. 


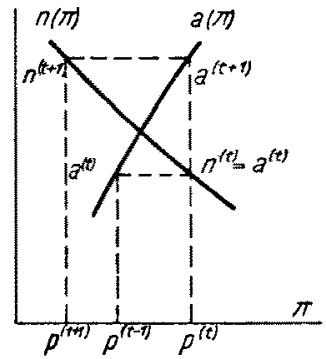

Abb. 2

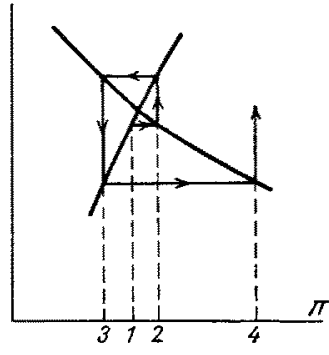

Abb. 2 a

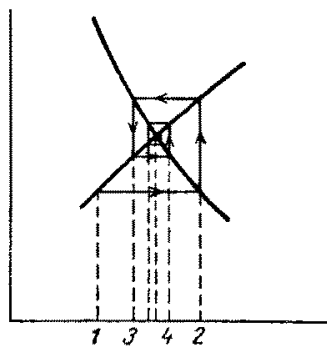

$\mathrm{Abb}, 2 \mathrm{~b}$

Ein wirkliches Beispiel eines solchen Falles ist der von Hanau gefundene Zusammenhang zwischen Schweineangebot und -preisen ${ }^{\mathbf{1}}$ ).

§ 3. Als dritten Fall kann man sich denjenigen denken, wo $a$ und $n$ beide noch einen Parameter enthalten, dessen Zeitablauf bekannt ist:

$$
a=a_{0}+a_{1} \pi+a_{2} A \quad n=n_{0}+n_{1} \pi+n_{2} N
$$

In diesem Falle lassen sich sowohl Nachfrage- wie auch Angebotskurve bestimmen. Es ist nämlich:

also

$$
\begin{gathered}
a_{1} p+a_{2} A=n_{1} p+n_{2} N, \\
p=\frac{a_{2} A-n_{2} N}{n_{1}-a_{1}} \text { und } u=n_{0}+\frac{n_{1} a_{2} A-n_{2} a_{1} N}{n_{1}-a_{1}} .
\end{gathered}
$$

Die statistische Analyse von $p$ und $u$ liefert nun die vier Koeffizienten $\frac{a_{2}}{n_{1}-a_{1}}, \frac{-n_{2}}{n_{1}-a_{1}}, \frac{n_{1} a_{2}}{n_{1}-a_{1}}$ und $\frac{-n_{2} a_{1}}{n_{1}-a_{1}}$, welche den Aufbau der $p *$ und der $u$-Schwankungen bestimmen, und aus diesen vier Koeffizienten lassen sich $a_{1}, a_{2}, n_{1}$ und $n_{2}$ ableiten. Man kann in diesem Fall auch den direkten Weg wählen und die Schwankungen von $u$ aufzubauen versuchen: das eine Mal aus denen von $p$ und $A$, woraus man die Koeffizienten $a_{1}$ und $a_{2}$ findet; das andere $\mathrm{Mal}$ aus denen von $p$ und $N$, woraus man $n_{1}$ und $n_{2}$ ableitet.

$\mathrm{Zu}$ dieser Kategorie gehört der kürzlich von mir veröffentlichte Fall des Kartoffelmehles ${ }^{2}$ ).

Zur Erläuterung der folgenden Ausführungen möge die Sachlage noch kurz wiedergegeben werden. Die Weltmarktversorgung mit Kartoffelmehl geschieht hauptsächlich durch Holland: In den Jahren 1922 bis 1928 wurden $77 \%$ der Weltausfuhr von Holland produziert. Die Untersuchung sah daher von der übrigen Produktion ab. Als Faktor $A$ wurde die holländische Produktion + Vorrat am Anfang des Produktionsjahres (1. September) gefunden. Diese Produktion ist weitgehend be-

1) Hanau: Die Prognose der Schweinepreise. Sonderheft 7 z. d. Vjh. zur Konjunkturforsch. 1928. Die Periode 1 ist hier $1^{1 / 2} \mathrm{Jahr}$.

2) Het verband tussehen den aardappeloogst en den prijs en den uitvoer van aardappelmeel. De Nederlandsehe Conjunctuur, S. 18. März 1930. Ich bin Herrn Dr. P. W. d e $L$ ang e zu vielem Danke verpflichtet für manche kritisehe Bemerkung bei der Bearbeitung. 
stimmt durch die Ernte an ,Fabrikskartoffeln", wie man sie in Holland von den "Konsumtionskartoffeln" unterscheidet. Als Faktor $N$ wurde die Kartoffelernte in Frankreich und Deutschland zusammen gefunden, welche die wichtigsten derjenigen Abnehmer sind, die selber auch Kartoffelmehl produzieren und deren Nachfrage von der eigenen Ernte nahezu bestimmt wird.

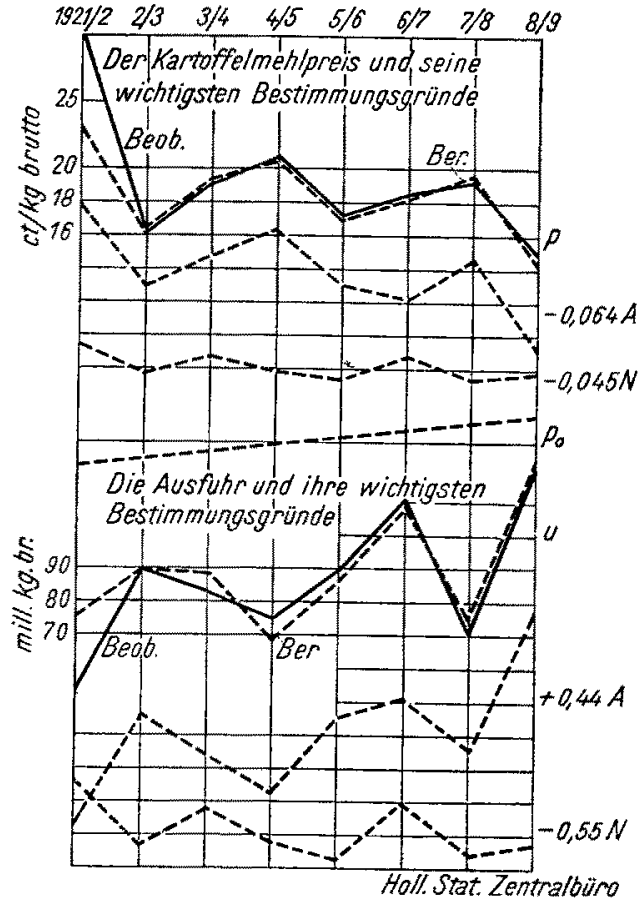

$\mathrm{Abb} \cdot 3$

Wählt man die folgenden Einheiten :

$a, n$ und $u$ in Millionen Kilogramm pro Produktionsjahr,

$\pi$ und $p$ in Gulden $/ 100 \mathrm{~kg}$ brutto), Albweichung vom „Normalpreis" $p_{0}{ }^{1}$ ),

$A$ in Millionen Kilogramm, Abweichung vom Mittelwert

$N$ in Prozentabweichung vom Mittelwert für $1922 / 23$ bis $1927 / 28$, so erhält man die mit Hilfe der statistisoh gefundenen Koeffizienten ,berechneten" Werte für $p$ und $u$ als

$p=-0,064 A-0,045 \mathrm{~N}$

$u=87+0,44 A-0,55 N$

Die Korrelationskoeffizienten der ,berechneten" und tatsächlichen $p$ bzw. $u$. Werte sind 1,00 bzw. 0,92 (siehe $\mathrm{Abb} .3)$.

In der angegebenen Weise

berechnet man hieraus die Nachfrage- und Angebotsfunktion als:

$$
n=87-6,9 p-0,86 N \quad a=87+12,2 p+1,22 \mathrm{~A} \text {. }
$$
Werte:

Direkte Berechnung (siehe oben) liefert die etwas verschiedenen

$$
n=87-6,2 p-0,85 N \quad a=87+11,2 p+1,18 \mathrm{~A}
$$

mit den Korrelationskoeffizienten 0,87 und 0,96 .

Wir legen unseren weiteren Berechnungen den Wert

$$
n=87-6,5 \pi-0,86 \mathrm{~N}
$$

der Nachfragefunktion zugrunde.

1) Dieser Normalpreis hat für die Produktionsjahre 1922/23 bis 1928/29 die Werte 17,$5 ; 17,9 ; 18,3 ; 18,7 ; 19,1 ; 19,5 ; 19,9$. Er besitzt also einen leichten Trend, der jedoch nicht den Trend des Preises darstellt, sondern ein Agglomerat von Trendlinien auch der übrigen Kurven und der Bequemlichkeit halber in dieser Weise zusammengefaßt gegeben worden ist. 
$\S 4$. Die Frage, womit wir uns nun weiter beschäftigen wollen, betrifft die Deutung der gefundenen Angebotskurve.

Im Gegensatz zur Deutung der Nachfragekurve, über deren Bedeutung man im allgemeinen einig ist und wobei man nicht viel anderes machen kann, als die gefundene Nachfragekurve als Faktum zu akzeptieren, gibt es bei der Deutung der Angebotskurve einige interessante Probleme.

Erstens ist der Charakter der Angebotskurve verschieden, je nachdem man es mit einem Markte zu tun hat, wo unbeschränkte Konkurrenz herrscht oder mit einem solchen, der mehr oder weniger monopolisiert ist. Im Fall eines Monopols besteht nach den bekannten Ausführungen der theoretischen Nationalökonomie sogar keine eigentliche unabhängige Angebotskurve im Sinne der ,einfachen statischen Theorie“; der Umsatz läßt sich bestimmen aus Nachfrage- und Kostenfunktion.

Zweitens kann man sich mit Recht fragen, ob eine statische Deutung des Angebots überhaupt zur Erklärung des wirklichen Sachverhaltes ausreichen wird. Der Einfluß essentiell dynamischer Elemente in der Preis- und Umsatzgestaltung ist seit dem Aufblühen der Konjunkturforschung nur allzu bekannt.

Daß solche Fragen bei der Deutung der Nachfragekurven - speziell von Nicht-Produktionsmitteln - fast keine Rolle zu spielen scheinen, braucht nicht zu wundern. Die durch die statische Theorie suggerierte Symmetrie von Angebot und Nachfrage ist doch in der ökonomischen Realität an wichtigen Punkten durchbrochen. Eine allgemeine Erklärung dieses Faktums dürfte im Umstand gelegen sein, daß jedes ökonomische Subjekt an vielen Märkten nachfragt, aber nur an wenigen anbietet, und daß sein Bestehen viel mehr vom Angebotemarkt abhängt als von den Nachfragemärkten. Dadurch ist schon deutlich, daß erstens allerhand Monopolbestrebungen für das Angebot viel mehr in Betracht kommen und zweitens auch das Blicken in die Zukunft - notwendige Bedingung für Dynamik - hier mehr gesucht werden muß als bei der Nachfrage.

Im folgenden wird versucht, $\mathrm{zu}$ bestimmen, welche von den beim jetzigen Stand der Theorie möglichen Deutungen die beste sei. Eine Wahl ist dadurch möglich, daß die durch jede Deutung verlangten $\mathrm{Zu}$ sammenhänge innerhalb gewisser Grenzen kontrolliert werden können, wie im folgenden noch gezeigt werden soll. Neben den schon genannten, ziemlich exakt meßbaren Größen, sind es auch noch einige weniger exakt zu fassende Daten, die das ermöglichen. Diese Daten sind folgende:

a) Die Struktur der betreffenden Industrie. Es gibt zwei Arten von Unternehmen in diesem $Z$ weige, nämlich kooperative und private. Von den kooperativen (Bauern-) Fabriken ist die große Mehrheit in einem Verkaufsbüro organisiert, das wir mit I bezeichnen wollen. Weitere drei sind unabhängig (II, III, IV). Von den privaten Fabriken ist eine (V) die weitaus größte, eine zweite (VI) hat auch noch Bedeutung. Die übrigen vier sind viel kleiner. Zur Beleuchtung der relativen Wichtigkeit dieser Betriebe mögen die Produktionszahlen für 
1926/27 und 1927/28 (ein reiches und ein armes Kartoffeljahr) gegeben werden ${ }^{1}$ ):

$\begin{array}{ccccccc} & \text { I } & \text { II } & \text { III } & \text { IV } & \text { V } & \text { VI } \\ 1926 / 27 & 470 & 66 & 44 & 35 & 48 & 25 \\ 1927 / 28 & 399 & 62 & 46 & 29 & 38 & 16\end{array}$

b) Die mittleren Produktionskosten. Wie man durch Vergleich der Mehl- und der Kartoffelpreise leicht erkennt, wird der wichtigste Teil der Produktionskosten durch die Kosten der Kartoffeln gebildet. Die jährlichen Schwankungen werden dann auch in der Hauptsache durch die Kartoffelpreise bestimmt sein. Der Jahresdurchschnitt wird aber höher sein, obgleich natürlich nicht höher als die Mehlpreise. Innerhalb gewisser Grenzen kann man sich also eine Idee über die Kosten bilden. Allerdings gilt dieses nur für die proportionalen Kosten. Wie aus dem folgenden auch noch ersichtlich sein wird, sind aber nur diese für uns wichtig. Die fixen Kosten spielen - wie z. B. auch nach Clark - keine Rolle bei kurzdauernden Schwankungen.

Zur Beurteilung der Kartoffelpreise nahmen wir die mittleren Auszahlungen der kooperativen Fabriken an ihre Mitglieder für eine Menge Kartoffeln, welche zur Herstellung von $1 \mathrm{~kg}$ Mehl benutzt werden. Zusammen mit den übrigen Utberlegungen führten sie zu den folgenden Angaben über die proportionalen Kosten: Sind diese

$$
\text { so ist } \quad 14<k_{0}<17 \quad k=k_{0}+k_{1} A .0,05<-k_{1}<0,08 \text {. }
$$

c) Die Verteilung der Kapazität über die verschiedenen Produktionskosten. Hierüber kann man sich eine ganz rohe Idee bilden, wenn man berücksichtigt, daß die Auszahlungen der verschiedenen kooperativen Fabriken im allgemeinen nicht mehr als 3 ct Unterschied zeigen. Das könnte nämlich darauf hinweisen, daß es einen Produktionskostenunterschied von dieser Größenordnung gibt, und der, sagen wir, höchstens 5 ct beträgt.

$\S 5$. Deutung 1: Statische Konkurrenz. Nach der einfachen, statischen Theorie des Marktes mit unbeschränkter Konkurrenz, geht die Angebotskurve offenbar parallel mit der soeben genannten Verteilung der Produktionskapazität über die verschiedenen Produktionskosten, d. h. mit der Linie, welche bei jedem Preis angibt, wieviel für diesen Preis ohne Verlust produziert werden kann ${ }^{2}$ ). Würde das mit unserer

1) Entnommen dem ,"Verslag van de Kamer van Koophandel en Fabrieken voor de Veenkolonien te Veendam". 1926 u. 1927.

2) Das gilt jedenfalls solange, man, was meistens stillschweigend geschieht, annimmt, daß jede Fabrik eine bestimmte Kapazität hat und dem Gesetz der zunehmenden Mehrerträge gehorcht. Das Angebot im Walrasschen oder $\mathbf{E d g e w}$ or th schen Sinn ist dann entweder Null oder die volle Kapazität, je nachdem der Preis unter oder über dem Produktionspreis liegt. Das Gesamtangebot ist dann eine treppenartige Kurve, welehe um die Kapazität eines Unternehmens steigt, sobald der Produktionspreis für dieses Unternehmen überschritten ist. Für jeden Produktionspreis $k(\mathrm{Abb} .4 \mathrm{a})$ ist das Angebot eigentlich innerhalb gewisser Grenzen unbestimmt. 
Angebotskurve der Fall sein und würde Extrapolation dieser Kurve bis $a=o$ einerseits und $a=120$ (Maximumwert von $u$ ) anderseits gestattet sein, so könnte man einen Vergleich machen. Diese Extrapolation liefert statt der obengenannten 5 ct: $\frac{120}{12}=10$, was offenbar zu groß wäre. Es ist aber durchaus fraglich, ob eine solche Extrapolation gestattet ist, und in diesem Sachverhalt ist dann auch kein Kriterium über die $\mathrm{Zu}$ lässigkeit dieser Deutung zu sehen.

Ein für diese Deutung weniger günstiger Umstand scheint mir zu sein, daß tatsächlich auch diejenigen Fabriken produzieren, die nach der statischen Theorie nicht produzieren sollten; überhaupt ist das Auftreten eines so großen Vorrats, wie es in einzelnen Jahren der Fall ist $^{1}$ ), kaum $\mathrm{zu}$ verstehen, wenn man diese Theorie anwendet. Sehr zu verwundern braucht man sich darüber meines Erachtens nicht; das ,auf die Dauer..." womit die Theoreme der Statik anfangen, ist in einem Jahre sehr wahrscheinlich nicht erreicht (vgl. auch § 7).

§ 6. Deutung 2: Statisches Monopol. Das soeben über die Statik Gesagte trifft für den Fall des Monopols viel weniger zu wie für den Konkurrenzfall, wie man sich leicht klar macht. Es hat deshalb meines Erachtens noch sehr wohl Zweek, diesen Fall näher zu betrachten. Die Kontrollmöglichkeiten sind hier wesentlich größer, da eine unabhängige Angebotskurve hier nicht besteht, sondern das Angebot sich nach der Nachfrage im Zusammenhang mit den Kosten regelt. Wir wollen jetzt in der Weise vorgehen, daß wir die Nachfragefunktion als durch (3) gegeben betrachten und daraus mit Hilfe einer vorläufig in der unbestimmten Form (4) gehaltenen Kostenfunktion den Preis $p$ bestimmen. Durch Gegenüberstellung des so gefundenen $p$ mit dem experimentellen (2) läßt sich dann eine Kontrolle machen. Es ist dabei nicht notwendig, auch den Umsatz $u$ zu berechnen und mit (2) zu vergleichen, da wegen des funktionellen Zusammenhanges dieser Vergleich nichts Neues mehr ergeben kann.

Bekanntlich wird nun der Monopolpreis $p$ dadurch bestimmt, daß der zu erzielende Gewinn

$n\left(p+p_{0}-k_{0}-k_{1} A\right)-K=\left(n_{0}+n_{1} p+n_{2} N\right)\left(p+p_{0}-k_{0}-k_{1} A\right)-K$ wo $K$ die fixen Kosten sind, ein Maximum wird. Das ist der Fall für

$$
p=-\frac{n_{0}+n_{2} N}{2 n_{1}}+\frac{1}{2}\left(k_{0}+k_{1} A-p_{0}\right)
$$

was mit den bekannten Werten von $n_{0}, n_{1}, n_{2}$ und $p_{0}$ übergeht in:

$$
p=\left(-2,5+\frac{1}{2} k_{0}\right)-0,066 N+\frac{1}{2} k_{1} A \text {. }
$$

Der Vergleich mit (2): $p=-0,045 N-0,064 A$ gibt Anlaß zu den folgenden Bemerkungen. Die U̇bereinstimmung der Koeffizienten für $N$ ist nicht ungünstig, wenn man bedenkt, daß, wie aus $\mathrm{Abb} .3$ - wo die Glieder der Gleichungen (2) in vergleichbarem Maßstab angegeben

1) Der Vorrat am 1. September schwankte 1922 bis 1928 zwischen 0 und 40 Millionen Kilogramm. 
sind - ersichtlich ist, der Einfluß von $N$ auf den Preis nicht so stark ist. Identifizierung der übrigen Koeffizienten liefert jedoch

$$
k_{0}=5 \quad k_{1}=-0,13,
$$

was entschieden unbrauchbare Werte sind. Für mehrere Jahre würden die Kosten sogar negativ ausfallen.

\$ 7. Deutung 3: Statische, beschränkte Konkurrenz. Die Preisbildung in den Zwischenstufen zwischen einem Monopol und der Konkurrenz sehr vieler Verkäufer, deren keiner den Preis zu beeinflussen vermag, gehört leider zu den umstrittenen Gebieten der theoretischen Nationalökonomie. Die einzige Formel, die, soweit mir bekannt ist, für diese Fälle besteht, die von Cournot, wird z. B. von Edgeworth $^{1}$ ) absolut abgelehnt. Dagegen wird sie warm verteidigt von Wicksell und Schumpeter ${ }^{2}$ ). Mindestens scheint mir diese Formel eine beschränkte Gültigkeit zu haben, wie das Chamberlin ${ }^{3}$ ) darstellt. Im folgenden soll daher auch diese Formel als Deutungsschema unserer Angebotskurve benutzt werden. Das Schöne an dieser Formel ist, daß bei der Form der Kostenfunktion (4), die Zahl $\mu$ der Konkurrenten explizite in ihr vorkommt, wodurch aus einer allgemeinen Formel alle Fälle (auch der des Monopols) durch Spezialwahl des $\mu$ hervorgehen.

Nach der Cournotschen Theorie ist nun

$$
n+\mu\left(p+p_{0}-k_{0}-k_{1} A\right) \frac{d n}{d p}=0
$$

wo jetzt $k_{0}$ und $k_{1}$ die arithmetischen Mittel der $k_{0}$ und $k_{1}$ aller Konkurrenten sind. Das gibt für $p$ :

$$
p=\frac{1}{1+\mu}(+13,4-0,132 N)+\frac{\mu}{\mu+1}\left(-18,5+k_{0}+k_{1} A\right) \text {. }
$$

Gibt $\operatorname{man} \mu$ die bzw. Werte $2,3,4, \infty$, so ergibt der Vergleich mit (2):

$\left.\begin{array}{rlrr}\mu & k_{0} & k_{1} & \text { Koeff. } v . N . \\ 2 & 12 & 0,10 & -0,044 \\ 3 & 14 & 0,09 & -0,033 \\ 4 & 15 & 0,08 & -0,026 \\ \infty & 18,5 & 0,07 & 0,000\end{array}\right\} \begin{gathered}\text { Koeff. } v . N . \\ \text { in }(2): \\ -0,045\end{gathered}$

Wie man sieht, ist eine ziemlich gute Ubereinstimmung $\mathrm{zu}$ bekommen für $\mu=3$ oder $\mu=4$. Das würde dann etwa bedeuten, daß die 3 bis 4 mächtigsten Exporteure den Preis in gegenseitiger Konkurrenz bestimmen. Wie man uber die Formel von Cournot auch denken mag, merkwürdig ist doch, daß sie in diesem Fall die einzige von den bekannten Theoremen $\mathrm{zu}$ sein scheint, welche uns helfen kann.

Der Fall $\mu=\infty$ ist offenbar gleichbedentend mit freier Konkurrenz, muß sich also mit dem Resultat der "einfachen statischen Theorie" decken. Obige Tabelle zeigt nun für $\mu=\infty$ keine Abuängigkeit des Preises von $N$,

1) Edgeworth: Papers Relating to Political Economy, Bd. I, S. $111 \mathrm{ff} .1925$.

2) Wicksell: Archiv f. Sozialwiss. u. Sozialpol., 58, S. 267. 1927; Schumpeter: Ebenda, S. 248.

3) Chamberlin: Quart. journ. of Econ, November 1929. S. 63. 


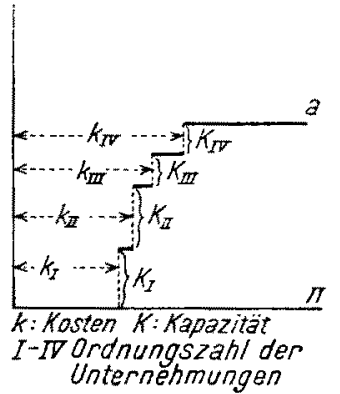

Abb, $4 \mathrm{a}$

Die Angebotskurve nach der ,einfachen statischen Theorie" nnter Ungleiche Kosten

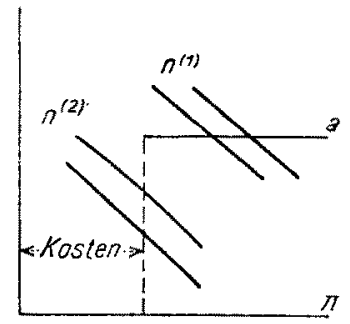

Abb. 4 b

Gleiche Kosten

d. h. also keine Preisänderung bei bloßer Nachfragenänderung; dieses tritt aber im einfachen statischen Schema nur dann auf, wenn die Angebotskurve vertikal verläuft, d. h. wenn das Angebot zwischen gewissen Grenzen unbestimmt ist. Nun ist in der Tat das Angebot prinzipiell unbestimmt, wenn der Preis den Kosten eines der konkurrierenden Firmen genau gleich ist (vergl. Abb. 4a). Diese Unbestimmtheit ist klein und unwichtig, solange jedes Unternehmen relativ klein ist und deshalb die prinzipiell diskontinuierliche Angebotskurve durch eine kontinnierliche ersetzt werden kann, wie meistens angenommen wird. Sie wird aber wichtig (Abb. 4b), falls die Produktionskosten der konkurrierenden Unternehmungen zusammenfallen, wie es hier im behandelten Fall nahezu zu sein scheint (vergl. §4). Es ist also kein Widerspruch zwischen den Resultaten dieser Theorien für den Grenzfall $\mu=\infty$; das Merkwürdige ist sogar, daß die ,einfache statische Theorie“" für diesen Fall eigentlich versagt, wenn $(\mathrm{Abb} .4 \mathrm{~b})$ die Nachfragekurve den Stand $n^{(2)}$ hat. Auf diese Probleme soll an dieser Stelle aber nicht weiter eingegangen werden.

\$ 8. Deutung 4: Dynamische Konkurrenz. Bevor wir schließlich auch noch einen solchen Deutungsversuch machen, wird eine kurze Darstellung dessen, was wir mit Dynamik meinen, am Platze sein.

Die Hauptzüge einer Dynamik scheinen mir folgende zu sein: Essentiell ist jetzt die Betrachtung einer Reihe von Wirtschaftsperioden (Zeiteinheiten), in welchen die ökonomischen Größen jedesmal einen anderen Wert haben können, den wir durch obere Indizes angeben. Am Anfang jeder Periode faßt jedes Subjekt Fntschlüsse bezüglich seines ökonomischen Handelns. Es bestimmt am Anfang der Periode $t$ sein Angebot oder seine Nachfrage an verschiedenen Märkten für die Perioden $t, t+1, t+2$ usw. Als Daten bei dieser Bestimmung gebraucht es teilweise Fakten, teilweise Erwartungen, z. B. den Preis wie er jetzt ist und den Preisablauf den es erwartet. Als Prinzip bei dieser Bestimmung gebraucht es das Maximieren seiner Ophelimitätsfunktion, wie es diese jetzt (am Anfang der Periode $t$ ) beurteilt. In dieser Ophelimitätsfunktion kommen alle die Größen, auch die künftigen, die eine Rolle spielen, vor. Der mit diesen Daten und nach diesem Prinzip 
gefaßte Entschluß bildet den Wirtschaftsplan zur Zeit $t$; er ist charakterisiert durch Reihen wie

$$
x^{(t)} x^{(t+1)} x^{(i+2)} \ldots \ldots
$$

wo $x$ eine Angebots- oder Nachfragemenge, eventuell einen Preis (beim Monopolisten) darstellt. Von diesem Plan sind nur ein Teil der Größen zur Realisierung vorbestimmt, nämlich alle diejenigen, die gleich in Taten umgesetzt werden müssen. Das sind die Mengen, die auf die erste Periode $(t)$ Beziehung haben bzw. diejenigén, die sich auf spätere Perioden beziehen, insoweit diese durch Verträge usw. schon jetzt festgesetzt werden müssen.

Am Anfang der Periode $t+1$ wird in genau derselben Weise ein Plan festgestellt, worin teilweise die im Plan $(t)$ bestimmten Größen als Daten auftreten (nur solche, die schon realisiert worden sind) und andere dagegen "revidiert" werden. Auch von diesem Plan wird ein bestimmter Teil realisiert. Das System aller so „realisierter" Größen bildet den dynamischen Wirtschaftsablauf ${ }^{1}$ ).

§ 9. Diesen Gedankengang wollen wir auf unser Beispiel in sehr bescheidenem Maß anwenden, wobei eventuelle Undeutlichkeiten möglichst noch geklärt werden.

Die folgende Überlegung kann gewissermaßen diese Anwendung motivieren. Wenn nach günstiger Ernte eine übernormale Menge Kartoffelmehl produziert worden ist, so bemerkt man, da $B$, trotz des Bestehens konkurrierender Produzenten, der Preis doch nicht so niedrig wird, daB die ganze produzierte Menge verkauft wird. Dies kann dadurch erklärt werden, daß die Produzenten einen Teil ihrer Produktion fürs nächste Jahr aufgespeichert behalten, in der Erwartung, daß der Preis sich dann für sie günstiger gestalten werde. Offenbar hat man es dann mit einer dynamischen Angebotsbildung zu tun.

Im einfachsten Fall könnte man den Preis folgendermaßen bestimmt denken: Nur ein weiteres Produktionsjahr wird mit in die Berechnungen einbezogen. Man erwarte für dieses zweite Jahr eine normale Ernte und also normale Mehlproduktion und gleichfalls eine normale Nachfrage. Diese normale Produktion ist gleich $a_{0}$; die normale Nachfrage ist gleich

$$
n_{0}+n_{1} p^{(2)}
$$

(der abnormale Teil $n_{2} N$ ist fortgefallen, $p^{(2)}$ ist der Preis im zweiten Jahre). Die verkauften Mengen in den zwei Jahren zusammen werden wegen der Konkurrenz gleich den produzierten $\left.\operatorname{sein}^{2}\right)$, die Verteilung über die zwei Jahre wird aber so gewählt (durch Angebotsrückhaltung

1) Vieles von den hier entwickelten Gedanken verdanke ich den anregenden Diskussionen mit Herrn Prof. Dr. P. Ehrenfest vom Institut für theoretische Physik der Leidener Universität.

2) Abgesehen von einer ,normalen“ Vorratsbildung, welche wir gleich der mittleren annehmen. 
in einem Uberflußjahr und umgekehrt), daß der in diesen Umständen mögliche Gewinn

$$
\left(p^{(1)}+p_{0}-k^{(1)}\right)\left(n_{0}+n_{1} p^{(1)}+n_{2} N^{(1)}\right)+\left(p^{(2)}+p_{0}-k^{(2)}\right)\left(n_{0}+n_{1} p^{(2)}\right)
$$

zum Maximum wird. Wir haben es also mit einem Maximumproblem mit der Nebenbedingung

zu tun.

$$
n_{0}+n_{1} p^{(1)}+n_{2} N^{(1)}+n_{0}+n_{1} p^{(2)}=\left(a_{0}+A^{(1)}\right)+\left(a_{0}\right)
$$

Nur der resultierende Preis $p^{(1)}$ interessiert uns nun, weil nur dieser - und die dadurch bestimmte Nachfrage - „realisiert" wird. Wir finden:

$$
\begin{aligned}
& p^{(1)}=\left(\frac{1}{4} k_{1}+\frac{1}{2 n_{1}}\right) A^{(1)}-\frac{3 n_{2}}{4 n_{1}} N^{(1)} \\
& =\left(\frac{1}{4} k_{1}-0,077\right) A^{(1)}-0,10 N^{(1)} .
\end{aligned}
$$

Ein Vergleich mit (2) zeigt, daß die Nachfrageabhängigkeit jetzt etwas zu stark geworden ist, während man für $k_{1}$ jetzt einen positiven Wert finden würde, was offenbar kein schönes Resultat ist.

Die Folgerung scheint also nahezuliegen, da $B$ von den versuchten Deutungen diejenige mit der Cournotschen Formel der beschränkten Konkurrenz die beste sei. Da in diesem Gebiete aber die äuerste Vorsicht geboten ist, schien es mir erwünseht, auch die negativen Resultate mitzuteilen.

\$10. Die Frage liegt schließlich nahe, ob es auch eine dynamisohe Form (im obigen Sinne) der Monopolgleichungen und der Cournotschen Gleichungen gebe. Soweit ich sehe, gibt es eine solche nioht: sie fällt entzwei in zwei statische Gleichungssysteme. Das würde damit zusammenhängen, da $B$ wenigstens ein Monopolist ,aus der augenblicklichen Lage herausholt, was herauszuholen ist" und deshalb von einer Verschiebung in die Zeit nichts zu erwarten hat. Demgegenüber fühlt der in freier Konkurrenz befindliche Verkäufer den Preis als etwas gegebenes, und für ihn hat es sehr wohl Zweck, sein Angebot zu verschieben.

Auf die vielen interessanten Probleme, welche die so aufgefaßte Dynamik in Aussicht stellt, sei hier nicht weiter eingegangen; es würde zu weit vom Ziele führen. 